\title{
Effect of High-Flow Nasal Cannula Oxygen Therapy in Immunocompromised Subjects With Acute Respiratory Failure
}

\author{
Hanyujie Kang, Zhiling Zhao, and Zhaohui Tong
}

\begin{abstract}
BACKGROUND: Various studies have been performed to examine the effect of high-flow nasal cannula (HFNC) in immunocompromised patients with acute respiratory failure (ARF). However, the results were inconsistent. Thus, we conducted a meta-analysis to evaluate the effect of HFNC oxygen therapy in immunocompromised patients with ARF versus conventional oxygen therapy and noninvasive ventilation (NIV). METHODS: Relevant studies published prior to May 11, 2019, were systematically searched. The primary outcome was intubation rate; secondary outcomes were mortality (ICU mortality, in-hospital mortality, and 90-d mortality) and ICU-acquired infections. Data were pooled using the random effects model. RESULTS: Of 832 identified studies, 8 were eligible for inclusion in our analysis $(N=2,167$ subjects). HFNC was associated with lower intubation rates compared to conventional oxygen therapy (risk ratio [RR] 0.89, 95\% CI 0.79-1.00, $P=.040$ ), but we found no significant difference in the rate between HFNC and NIV (RR 0.74, 95\% CI 0.46-1.19, $P=.22$ ). We also found that HFNC did not increase the risk of ICU-acquired infections (RR 0.86, 95\% CI 0.63-1.18, $P=.35$ ). However, in comparison to other noninvasive therapies, HFNC exhibited no differences in ICU mortality (RR 0.82, 95\% CI 0.58-1.17, $P=.28$ ), in-hospital mortality (RR $0.92,95 \%$ CI $0.74-1.15, P=.48$ ), or 90-d mortality (RR $0.98,95 \%$ CI 0.81-1.18, $P=.82$ ). CONCLUSIONS: Our results suggest that HFNC may be a feasible alternative to NIV, with lower intubation rates and no increased risk for ICU-acquired infections compared to standard oxygen therapy. However, HFNC did not appear to reduce mortality in immunocompromised subjects with ARF compared with other noninvasive therapies. Further high-quality randomized controlled trials should be performed to confirm these findings. Key words: high-flow nasal cannula oxygen therapy; conventional oxygen therapy; noninvasive ventilation; acute respiratory failure; immunocompromised host; intubation rate. [Respir Care 2020;65(3):369-376. (C) 2020 Daedalus Enterprises]
\end{abstract}

\section{Introduction}

Acute respiratory failure (ARF) is a life-threatening complication that affects immunocompromised patients, ${ }^{1}$ ac-

\footnotetext{
The authors are affiliated with the Department of Respiratory and Critical Care Medicine, Beijing Institute of Respiratory Medicine, Beijing ChaoYang Hospital, Capital Medical University, Beijing, China.

Drs Kang and Zhao are co-first authors.

Dr Tong is supported in part by the Capital's Funds for Health Improvement and Research (2016-1-1061), Beijing Municipal Administration of Hospitals Clinical Medicine Development of Special Funding Support (ZYLX201312), and Beijing Municipal Administration of Hospitals' Ascent Plan (DFL20150302). The other authors have disclosed no conflicts of interest.
}

counting for $62.5 \%$ of ICU admissions. ${ }^{2}$ Those who require invasive mechanical ventilation exhibit higher mortality, ${ }^{3-5}$ so it is imperative to avoid intubation. Several noninvasive respiratory support methods are available, such as noninvasive ventilation (NIV) and conventional oxygen

\footnotetext{
Supplementary material related to this paper is available at http:// www.rcjournal.com.

Correspondence: Zhaohui Tong MD PhD, Department of Respiratory and Critical Care Medicine, Beijing Institute of Respiratory Medicine, Beijing Chao-yang Hospital, Capital Medical University, 8 Gong Ti South Road, Chao-Yang District, Beijing, 100020, China. E-mail: tongzhaohuicy@sina.com.
}

DOI: $10.4187 /$ respcare.07205 
therapy ${ }^{6-8} \mathrm{NIV}$ is often ordered as the first-line ventilation strategy in immunocompromised patients with ARF. ${ }^{9}$ However, in a high-quality randomized controlled trial (RCT), early NIV therapy did not improve clinical outcomes compared with conventional oxygen. ${ }^{10}$ The best ventilatory management in this population to prevent intubation is still unclear.

In recent years, high-flow nasal cannula (HFNC) oxygen therapy has been widely applied in patients experiencing acute hypoxemic respiratory failure, exacerbation of COPD, postextubation, preintubation oxygenation, sleep apnea, cardiogenic pulmonary edema, and conditions entailing do-not-intubate orders. ${ }^{11,12}$ A 2015 study reported that HFNC improved the survival rate among subjects with acute hypoxemic respiratory failure, without impacting intubation rates, compared to standard oxygen therapy or NIV. ${ }^{13}$ HFNC refers to a technique that delivers heated and humidified oxygen at a high flow of up to $60 \mathrm{~L} / \mathrm{min}$ through a nasal cannula. ${ }^{12}$ This technique has some beneficial physiologic effects, including generating PEEP, maintaining mucociliary clearance function, flushing anatomical dead space, and reducing inspiratory effort. ${ }^{14,15}$ Thus, HFNC may help improve clinical outcomes.

Because of its physiologic efficacy and better tolerance, HFNC has received a great deal of attention in the treatment of immunocompromised patients with ARF, and HFNC is a good alternative to NIV or conventional oxygen therapy to prevent intubation. ${ }^{16,17}$ Some systematic reviews and meta-analyses have reported that HFNC might decrease mortality and the use of invasive mechanical ventilation in immunocompromised subjects with ARF compared to other noninvasive oxygen strategies. ${ }^{18,19}$ However, a recent meta-analysis, ${ }^{20}$ which included a new large-scale trial, ${ }^{21}$ found no benefit of HFNC over conventional oxygen therapy on mortality in immunocompromised subjects. These findings were consistent with another recent meta-analysis ${ }^{22}$ that focused on a heterogeneous patient population, which included immunocompromised subjects. As a result, the application of HFNC remains controversial.

We systematically reviewed the current literature and performed a meta-analysis to assess whether HFNC can improve clinical outcomes of immunocompromised patients with ARF compared to conventional oxygen therapy or NIV.

\section{Methods}

\section{Search Strategy}

We systematically searched for relevant studies published prior to April 21, 2019 in PubMed, Embase, Web of Science, and the Cochrane Library. The search terms were as follows: ("high-flow nasal cannula oxygen" or "high- flow nasal cannula" or "HFNC" or "nasal high flow" or "humidified high-flow nasal cannula" or "high-flow nasal oxygen therapy" or "nasal high-flow oxygen" or "highflow nasal oxygen" or "humidified high-flow nasal cannula" or "humidified high-flow nasal oxygen" or "HHFNC" or "HHFN" or "high-flow oxygen therapy" or "nasal highflow oxygen therapy" or "oxygen therapy" or "oxygen nasal cannula") and ("acute respiratory failure" or "acute hypoxemic respiratory failure" or "critically ill" or "respiratory insufficiency" or "acute respiratory distress syndrome" or "ventilatory depression" or "dyspnea") and ("hematologic" or "hematological" or "transplant" or "tumor" or "cancer" or "immunosuppression" or "immunosuppressed" or "immunocompromised"). We applied no language restrictions and manually screened the reference lists from included studies to identify other relevant articles. Two reviewers (HK, ZZ) independently searched and evaluated the quality of the studies. Any disagreement was resolved by a third person. The PROSPERO registration number was CRD42019130563.

\section{Study Selection}

The inclusion criteria were adult immunocompromised subjects with ARF, studies that compared HFNC with NIV or conventional oxygen therapy, studies that reported the primary outcome of intubation rate or secondary outcomes (mortality, ICU-acquired infections) that could be directly extracted or calculated, and any observational studies or RCT studies.

The exclusion criteria were as follows: (1) reviews, metaanalyses, case reports, letters, expert opinions, or study protocols; (2) content unrelated to immunocompromised subjects with ARF or HFNC; (3) the targeted outcomes were not reported; (4) enrolled subjects were $<18$ y old or animal studies; (5) were not clinical trials; (6) were crossover trials; (7) were duplicates; (8) insufficient data; (9) experimental group did not receive HFNC; (10) control group did not receive NIV or conventional oxygen therapy; (11) use of HFNC in the postextubation or periprocedural setting; (12) multiple publication; or (13) abstract.

\section{Data Extraction and Risk of Bias Assessment}

Two reviewers (HK, ZZ) independently extracted data from included trials and assessed the risk of bias. Any disagreement was resolved by discussion. We extracted the following data from the eligible studies: first author, publication year, hospital environment, study design, criteria for enrollment, subject characteristics, total number of participants, intervention description, and outcomes. If any data were inadequate, we contacted the corresponding authors by e-mail. The risk of bias of the included RCTs was assessed with the Cochrane Collaboration Risk of 
Bias tool ${ }^{23}$ and rated as "low," "unclear," or "high" in several domains. The risk of bias of the included observational cohort studies was assessed with the NewcastleOttawa scale, which concentrated on 3 domains: selection, comparability, and outcome. A study could be awarded a maximum of 9 stars on items and classified as having a high risk (1-3 points), intermediate risk (4-5 points), or low risk of bias (6-9 points). ${ }^{24}$

\section{Outcomes}

The primary outcome was intubation rate. The secondary outcomes were mortality (ICU mortality, in-hospital mortality, and 90-d mortality) and ICU-acquired infections.

\section{Statistical Analysis}

We presented the risk ratios (RR) with 95\% CIs for dichotomous outcomes. Values for continuous outcomes were given as the mean \pm SD. Meta-analysis was performed using the Mantel-Haenszel random-effects model. Statistical heterogeneity across trials or subgroups underwent $\mathrm{I}^{2}$ testing, with values $>50 \%$ regarded as being indicative of moderate-to-high heterogeneity. ${ }^{25}$ Subgroup analyses and sensitivity analyses were used to explore the sources of heterogeneity. A prespecified subgroup analysis was conducted for the main outcomes (ie, studies that compared HFNC with NIV versus HFNC with conventional oxygen therapy). We also conducted sensitivity analyses by sequentially omitting one study each time or excluding nonrandomized studies to identify the potential influence. We evaluated the possibility of publication bias by constructing a funnel plot when $>10$ studies were included, and we defined significant publication bias as $P<.05$. Statistical analyses were conducted using Review Manager 5.2 (Cochrane IMS, Oxford, United Kingdom).

\section{Results}

\section{Search Results}

We identified 832 citations, of which 113 were duplicates (Supplementary Fig. 1; see the supplementary materials at http://www.rcjournal.com). After screening the titles and abstracts, 665 records were excluded because they did not meet the inclusion criteria. We reviewed 54 records in full, of which 46 were excluded for reasons described in the supplemental materials (at http://www. rcjournal.com). Ultimately, 8 studies involving a total of 2,167 subjects were included in the meta-analysis. ${ }^{1,21,26-31}$ All of the included studies received informed consent from each participant and were approved by an ethics committee or institutional review board.

\section{Study Characteristics and Risk of Bias}

The characteristics of the included trials are listed in the supplementary materials (see http://www.rcjournal.com). These studies were published between 2015 and 2018. The sample size varied from 38 to 836 participants. Two studies were RCTs, ${ }^{21,30}$ two were post hoc analysis of RCTs, ${ }^{27,29}$ and four were observational cohort studies.1,26,28,31 Two studies compared HFNC with both conventional oxygen therapy and NIV,1,29 two compared HFNC with NIV only, 26,28 and four compared HFNC with conventional oxygen therapy only. $21,27,30,31$ The assessment of risk of bias are shown in the supplementary materials (see http:// www.rcjournal.com). Four RCTs showed low risk of bias in nearly all of the items, however, two were post hoc analysis of RCTs. Four observational cohort studies were also of low risk of bias with 7-8 points assessed by the Newcastle-Ottawa scale. The publication bias was not assessed because of the limited number of included trials.

\section{Intubation Rate, Mortality and ICU-Acquired Infections}

Eight trials ${ }^{1,21,26-31}$ reported intubation rate (Fig. 1). The results suggested that HFNC was associated with a significant reduction in intubation rates compared to the control (RR $0.83,95 \%$ CI $0.72-0.97, P=.02$ ), with low heterogeneity among the studies $\left(\chi^{2}=9.95, \mathrm{I}^{2}=30 \%\right)$. Potential heterogeneity was explored with subgroup analysis (Supplementary Fig. 4; see the supplementary materials at http://www.rcjournal.com). The results indicated that HFNC was associated with a reduction of intubation rate in the subgroup comparing HFNC to conventional oxygen therapy (6 studies; RR $0.89,95 \%$ CI $0.79-1.00, P=.040$; $\mathrm{I}^{2}=3 \%$ ), but there was no difference between HFNC and NIV (3 studies; RR 0.74, 95\% CI 0.46-1.19, $P=.22$; $\mathrm{I}^{2}=67 \%$ ); the $P=.47$ for subgroup differences (comparing HFNC to conventional oxygen therapy or comparing HFNC to NIV). We conducted a sensitivity analysis (Supplementary Fig. 5; see the supplementary materials at http://www.rcjournal. com) by omitting observational trials. The results indicated HFNC could reduce intubation rates (4 studies; RR 0.87, 95\% CI $0.75-1.00, P=.050$ ), with no statistical heterogeneity $\left(\chi^{2}=1.22, \mathrm{I}^{2}=0 \%\right)$. Six studies $1,21,26,28,29,31$ assessed the effect of HFNC on ICU mortality (Fig. 2). There were no differences between HFNC and control group (RR $0.82,95 \%$ CI $0.58-1.17, P=.28$ ), with moderate heterogeneity $\left(\chi^{2}=15.15, \mathrm{I}^{2}=67 \%\right)$. We performed subgroup analyses and sensitivity analyses to explore the sources of heterogeneity. Pool analysis showed that HFNC did not reduce ICU mortality in comparison to conventional oxygen therapy (4 studies; RR $1.01,95 \%$ CI $0.74-1.36, P=.97 ; \mathrm{I}^{2}=59 \%$ ); the result was consistent in the subgroup comparing HFNC to NIV (3 studies; RR 


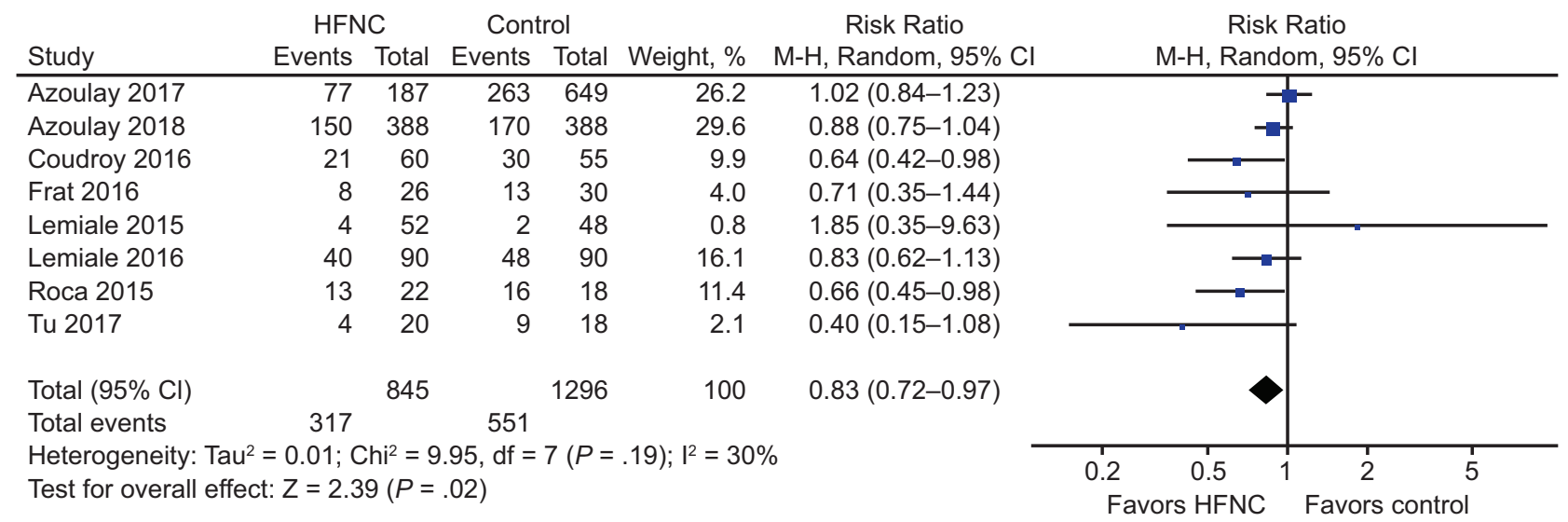

Fig. 1. Forest plot for intubation rate associated with high-flow nasal cannula (HFNC) in immunocompromised subjects with acute respiratory failure.

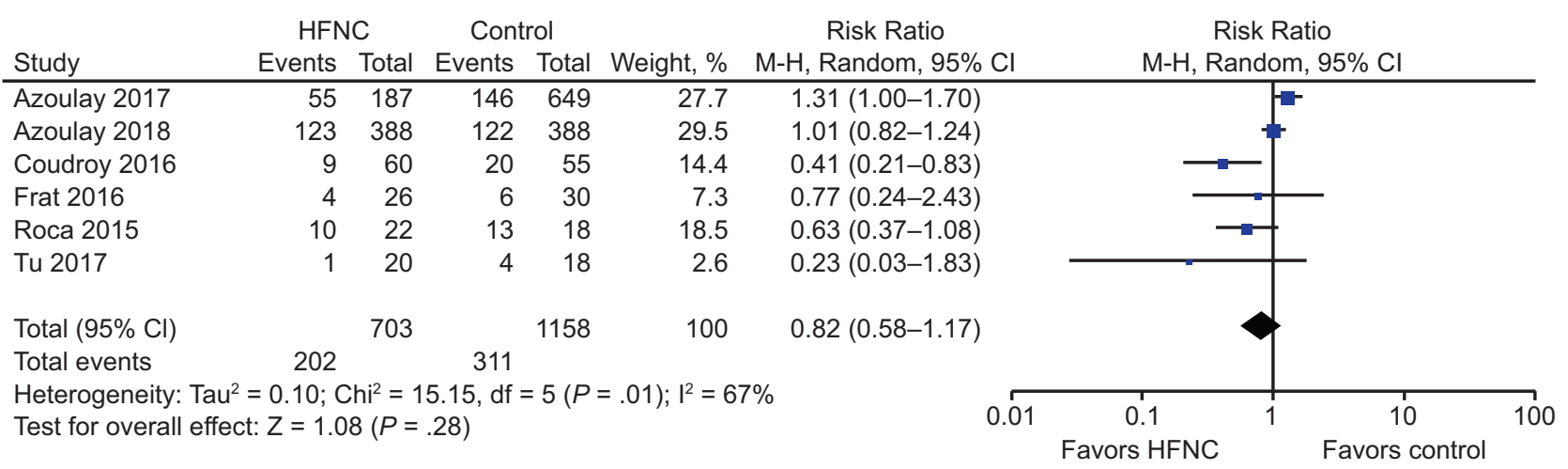

Fig. 2. Forest plot showing the effect of high-flow nasal cannula (HFNC) on ICU mortality of immunocompromised subjects with acute respiratory failure.

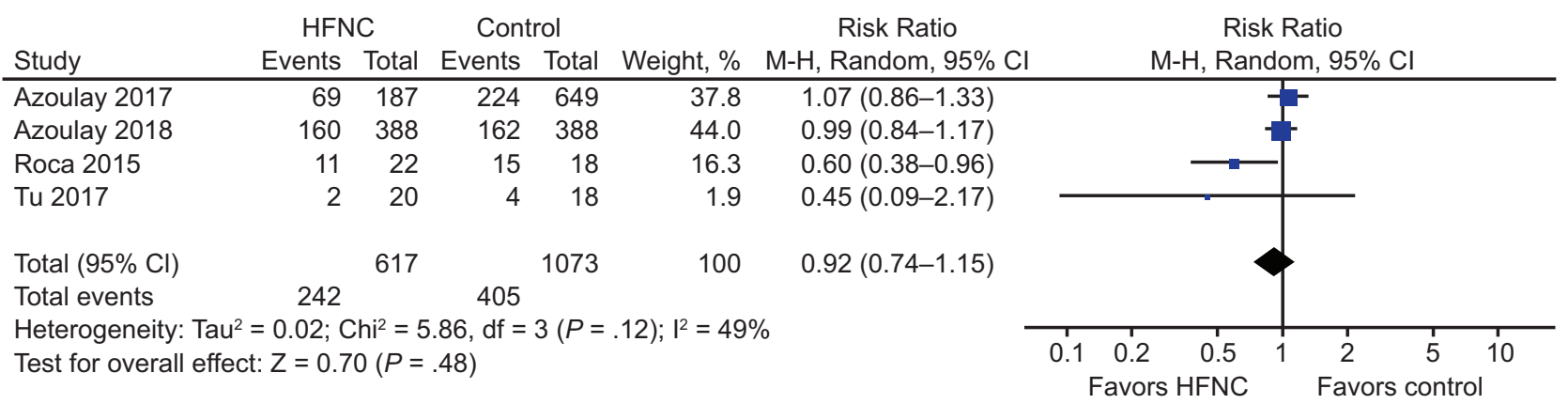

Fig. 3. Forest plot showing the effect of high-flow nasal cannula (HFNC) on in-hospital mortality of immunocompromised subjects with acute respiratory failure.

$0.61,95 \%$ CI $\left.0.24-1.56, P=.30 ; \mathrm{I}^{2}=76 \%\right)(P=.32$; see the supplementary materials at http://www.rcjournal.com). We then conducted sensitivity analysis to explore the potential heterogeneity by excluding nonrandomized studies (Supplementary Fig. 7; see the supplementary materials at http://www.rcjournal.com), and we found no differences in ICU mortality between the HFNC and control group (2 studies; RR 1.00, 95\% CI 0.82-1.23, $P>.99$ ), with no heterogeneity $\left(\chi^{2}=0.21, \mathrm{I}^{2}=0 \%\right.$ ). Four studies ${ }^{1,21,26,31}$ examined in-hospital mortality (Fig. 3), which did not show significant differences between the 2 groups (RR 0.92, 95\% CI $0.74-1.15, P=.48)$. There was mild heterogeneity $\left(\chi^{2}=5.86, \mathrm{I}^{2}=49 \%\right)$, and this was explored with subgroup analysis (Supplementary Fig. 8; see the supplementary materials at http://www.rcjournal.com). In the subgroup comparing HFNC to conventional oxygen therapy, 


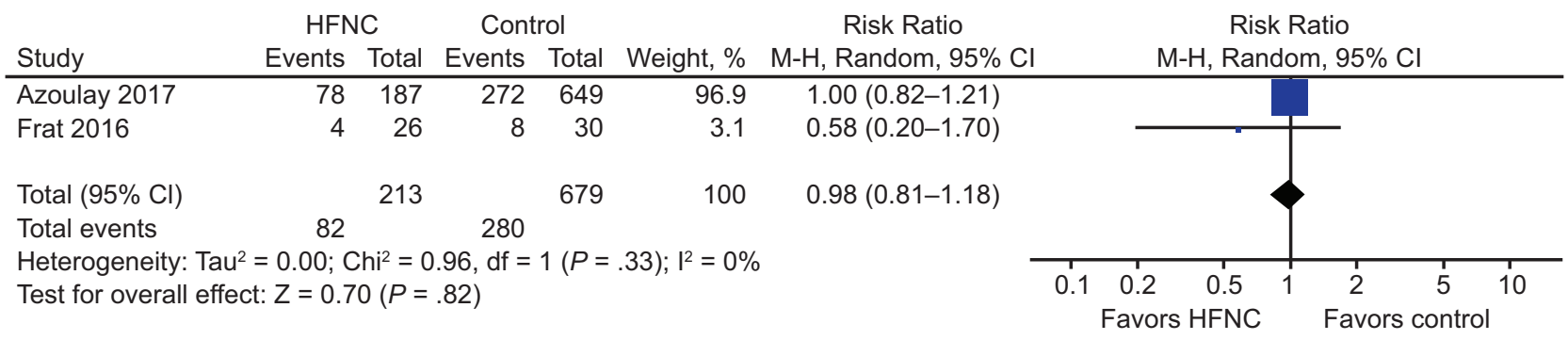

Fig. 4. Forest plot showing the effect of high-flow nasal cannula (HFNC) on 90-d mortality of immunocompromised subjects with acute respiratory failure.

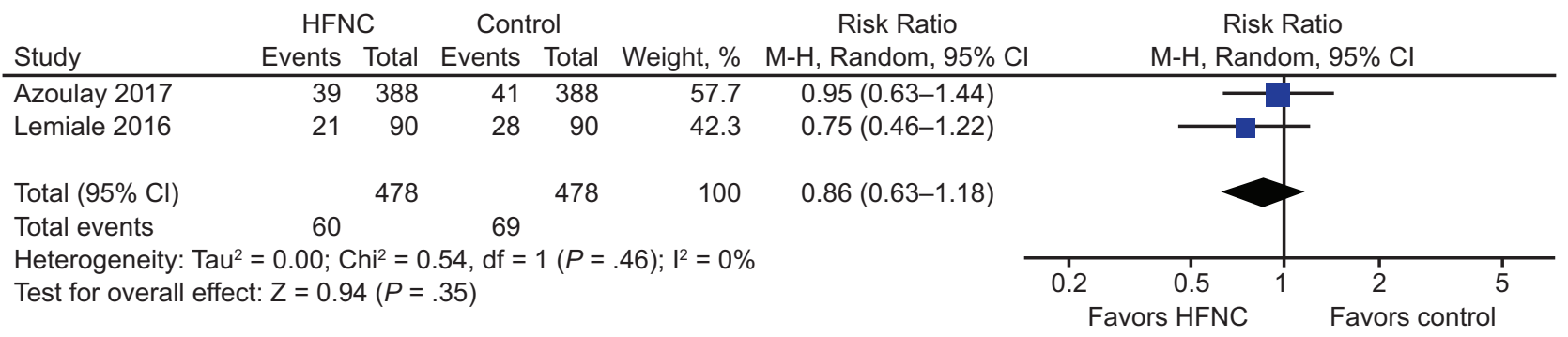

Fig. 5. Forest plot showing the effect of high-flow nasal cannula (HFNC) on ICU-acquired infections of immunocompromised subjects with acute respiratory failure.

there was no difference in hospital mortality (3 studies; RR $0.94,95 \%$ CI $0.73-1.22, P=.66 ; \mathrm{I}^{2}=66 \%$ ). In the subgroup comparing HFNC to NIV, the result was similar (2 studies; RR $0.89,95 \%$ CI $0.69-1.16, P=.40 ; \mathrm{I}^{2}=0 \%$ ) $(P=.76)$. We also conducted sensitivity analysis by excluding observational cohort studies (Supplementary Fig. 9; see the supplementary materials at http://www. rcjournal.com), and the result was consistent (1 study; RR $0.99,95 \%$ CI $0.84-1.17, P=.88$ ). Two studies ${ }^{1,29}$ assessed 90-d mortality (Fig. 4). There were no differences in 90-d mortality between the HFNC and control group (RR $0.98,95 \%$ CI $0.81-1.18, P=.82$ ), with no heterogeneity $\left(\chi^{2}=0.96, \mathrm{I}^{2}=0 \%\right)$. Subgroup analysis suggested that HFNC did not reduce 90-d mortality in comparison to conventional oxygen therapy ( 2 studies; RR $0.99,95 \%$ CI $\left.0.71-1.38, P=.96 ; \mathrm{I}^{2}=12 \%\right)$ or NIV (1 study; RR 0.86, 95\% CI 0.68-1.09, $P=.22$ ); subgroup differences $P=.50$ (Supplementary Fig. 10; see the supplementary materials at http://www.rcjournal.com). Only 2 trials $^{21,27}$ assessed ICU-acquired infections (Fig. 5). Use of HFNC had no pronounced effect on ICU-acquired infections (RR 0.86, 95\% CI 0.63-1.18, $P=.35$ ), with no heterogeneity $\left(\chi^{2}=0.54, \mathrm{I}^{2}=0 \%\right)$.

\section{Discussion}

ARF is the main reason for ICU admission in immunocompromised patients, and it is associated with a high mortality rate. ${ }^{32}$ HFNC is easy to apply for the clinical treatment of these patients, which may reduce work of breathing, the risk of lung injury, and, potentially, the need to intubate. ${ }^{16,33}$ However, compared with conventional oxygen therapy or NIV, the effect of HFNC is still controversial.

Our systematic review and meta-analysis indicated that the intubation rate was lower with HFNC than with conventional oxygen therapy, whereas no significant difference was found between HFNC and NIV. We also found that HFNC did not increase the risk of ICU-acquired infections, although there was no reduction in mortality rates compared to either control group. Overall, the results demonstrated that HFNC could be an alternative to NIV, with a lower intubation rate in immunocompromised subjects compared to conventional oxygen therapy. The results were consistent with other meta-analyses of all kinds of ARF patients, not just limited to immunosuppressed subjects. ${ }^{34,35}$

The lower intubation rate in the subgroup comparing HFNC to conventional oxygen therapy could be due to several reasons. First, HFNC's physiologic effect of reducing dead space contributes to better carbon dioxide clearance. ${ }^{36,37}$ Second, the heated and humidified gas can maintain functional mucociliary clearance to avoid mucosal injury and improve patient comfort. ${ }^{15}$ Third, the technique generates PEEP of 2-4 $\mathrm{cm} \mathrm{H}_{2} \mathrm{O}$, which keeps alveoli open and improves gas exchange. ${ }^{38,39}$ Finally, high inspiratory flow reduces inspiratory effort and resistance..$^{40}$ In summary, HFNC may reduce the need for intubation by increasing oxygenation and reducing inspiratory effort. ${ }^{41}$ 
Moreover, we found that HFNC did not increase ICUacquired infections compared with conventional oxygen therapy. Given that intubation increases the risk of severe complications such as ventilator-associated pneumonia in immunosuppressed patients, ${ }^{42}$ it is necessary to use noninvasive oxygen strategies to avoid intubation and reduce the potential for ICU-acquired infections.

Our results indicate that there was no significant difference in intubation rates between HFNC and NIV. We hypothesized that both HFNC and NIV have similar physiological characteristics, such as generating PEEP, preventing alveolar collapse, reducing dead space, and improving gas exchange. $37,43,44$

However, we found that HFNC did not reduce mortality rates compared with conventional oxygen therapy or NIV, possibly because many factors relate to mortality, including underlying immune status, which can affect patient outcomes. For one thing, the underlying immune status can affect patient outcomes. For example, patients who are frequently immunocompromised may be prone to a poor prognosis, and ${ }^{45}$ outcomes can be swayed to some extent by different causes of immunosuppression. For instance, some studies report lower mortality in transplant recipients compared to the cancer patients. ${ }^{1,31,32}$ Older age, higher Simplified Acute Physiology Score II, and worse organ function have been associated with higher mortality. ${ }^{45,46}$ HFNC, conventional oxygen therapy, and NIV each have their strengths, and outcomes probably depend not only on how oxygen is delivered, but also on underlying diseases and patients' response to different therapies. Therefore, other patient conditions should also be taken into consideration when choosing oxygen therapy (eg, $\mathrm{P}_{\mathrm{aCO}}$ levels, device tolerability, underlying disease, etc). Our findings were different from 2 meta-analyses published in 2018, which reported that HFNC could reduce mortality compared with conventional oxygen therapy or NIV.18,19 We attributed the difference to the fact that we were more strict in our study selection and we included a recent highquality, large-scale trial done by Azoulay et al. ${ }^{21}$ This RCT suggested that oxygenation strategies may not be the optimal method to improve survival in immunocompromised subjects with ARF. ${ }^{21}$ Therefore, assessment of patient comorbidities before HFNC application is extremely important, and it may reduce mortality rates.

NIV is commonly used in immunocompromised patients with ARF as the first-line ventilation strategy. ${ }^{9}$ In our meta-analysis, however, the impact of HFNC on outcomes was similar to NIV. Moreover, using NIV with a face mask can generate high tidal volumes, which may result in ventilator-induced lung injury. ${ }^{47}$ HFNC also has other strengths compared to NIV, including easier application and an interface that does not affect eating and talking. ${ }^{47}$ HFNC also provides more precise $\mathrm{F}_{\mathrm{IO}_{2}}$ and low-level PEEP, which can improve oxygenation and may reduce lung injury, respectively. ${ }^{17}$

Potential heterogeneity could be detected in short-term mortality (ICU mortality and in-hospital mortality). Subgroup analysis was used to explore the source of heterogeneity. The results showed that subgroup analyses that compared HFNC with NIV or conventional oxygen therapy couldn't explain the heterogeneity. The possible reasons might be the small number of studies, included nonrandomized trials, and the risk of bias in some domains. Hence, we cannot confidently conclude that the effect of HFNC on short-term mortality was similar between subgroups. We performed sensitivity analyses by excluding any observational studies, and determined statistical heterogeneity could not be detected, with no change in our results. This finding suggests that the included non-randomized trials had potential impact on the outcomes and our results were reliable.

To our knowledge, this is the latest meta-analysis that evaluates the impact of HFNC on preventing intubation in immunocompromised subjects with ARF, comparing HFNC to conventional oxygen therapy or NIV. The results were partially consistent with a recently published systematic review ${ }^{20}$ that only compared HFNC with conventional oxygen therapy, without comparing HFNC with NIV.

Our review has the advantage of including more highquality, large-scale trials that focus on immunosuppressed subjects, with a comprehensive analysis comparing HFNC to both conventional oxygen therapy and NIV. Nevertheless, this meta-analysis has some limitations. First, the sample size was insufficient $(n<50)$ in 2 included trials. More large, high-quality studies are required in the future. Second, due to the limited number of relevant studies, observational cohort studies were also included in our metaanalysis, which may affect the quality of outcomes. However, we conducted sensitivity analyses by omitting these observational studies and found results consistent with previous research, suggesting that our findings are reliable. The low risk of bias of included observational studies may account for the result. Third, we did not explore the effect of HFNC on dyspnea, comfort, and breathing frequency because there were not enough trials and these were usually considered weaker outcomes. ${ }^{48}$ Future research should also focus on these important but easily overlooked outcomes. Finally, given the lack of available individual subject data, we were unable to assess the effect of immune status, underlying comorbidity, severity of disease, and age on subjects' clinical outcomes. Future research should concentrate more on these individual subjects' characteristics.

Although our study did not support the view that HFNC could reduce mortality, it can be considered as an alternative noninvasive support for immunocompromised patients with ARF to potentially avoid premature intubation. The 
only concern was that HFNC may lead to unnecessary delays in intubation. ${ }^{12}$ Because delayed intubation is associated with increased mortality in patients treated with HFNC, ${ }^{49}$ closely monitoring patients' responses to HFNC is important. Many indicators can predict the risk of HFNC failure at an early stage, such as continued tachypnea, poor oxygenation, and persisting thoracoabdominal asynchrony. ${ }^{50}$ Monitoring patient response to HFNC from the beginning is conducive to individualized treatment. If gas exchange and breathing frequency are not obviously improved within a few hours, intubation should be initiated as soon as necessary. ${ }^{51}$

\section{Conclusions}

HFNC may be a feasible alternative to NIV for reducing intubation rates compared to conventional oxygen therapy, without detrimental effects on ICU-acquired infections. However, compared with other noninvasive oxygen therapies, HFNC did not reduce mortality in immunocompromised subjects with ARF. The choice of the 3 types of noninvasive oxygen therapies should also be based on the underlying disease and patients' response to therapy. If necessary, intubation should be initiated without delay. Taken together, better patient selection, improvement in noninvasive oxygen strategies, and earlier recognition of HFNC failure may improve patient prognosis. Because there are some limitations in our meta-analysis, further high-quality RCTs should be performed to confirm the effects of HFNC on the immunocompromised population.

\section{REFERENCES}

1. Azoulay E, Pickkers P, Soares M, Perner A, Rello J, Bauer PR, et al. Acute hypoxemic respiratory failure in immunocompromised patients: the Efraim multinational prospective cohort study. Intensive Care Med 2017;43(12):1808-1819.

2. Azoulay E, Mokart D, Pene F, Lambert J, Kouatchet A, Mayaux J, et al. Outcomes of critically ill patients with hematologic malignancies: prospective multicenter data from France and Belgium-a groupe de recherche respiratoire en reanimation onco-hematologique study. J Clin Oncol 2013;31(22):2810-2818.

3. Esteban A, Frutos-Vivar F, Muriel A, Ferguson ND, Penuelas O, Abraira V, et al. Evolution of mortality over time in patients receiving mechanical ventilation. Am J Respir Crit Care Med 2013;188(2): 220-230.

4. Azoulay E, Lemiale V, Mokart D, Pene F, Kouatchet A, Perez P, et al. Acute respiratory distress syndrome in patients with malignancies. Intensive Care Med 2014;40(8):1106-1114.

5. Azevedo L, Caruso P, Silva U, Torelly AP, Silva E, Rezende E, et al. Outcomes for patients with cancer admitted to the ICU requiring ventilatory support: results from a prospective multicenter study. Chest 2014;146(2):257-266.

6. Rochwerg B, Brochard L, Elliott MW, Hess D, Hill NS, Nava S, et al. Official ERS/ATS clinical practice guidelines: noninvasive ventilation for acute respiratory failure. Eur Respir J 2017;50(2).

7. Faverio P, De Giacomi F, Sardella L, Fiorentino G, Carone M, Salerno F, et al. Management of acute respiratory failure in intersti- tial lung diseases: overview and clinical insights. BMC Pulm Med 2018;18.

8. Brambilla AM, Aliberti S, Prina E, Nicoli F, Del Forno M, Nava S, et al. Helmet CPAP vs. oxygen therapy in severe hypoxemic respiratory failure due to pneumonia. Intensive Care Med 2014;40(7): 942-949.

9. Cortegiani A, Madotto F, Gregoretti C, Bellani G, Laffey JG, Pham $\mathrm{T}$, et al. Immunocompromised patients with acute respiratory distress syndrome: secondary analysis of the LUNG SAFE database. Crit Care 2018;22(1):157.

10. Lemiale V, Mokart D, Resche-Rigon M, Pene F, Mayaux J, Faucher E, et al. Effect of noninvasive ventilation vs oxygen therapy on mortality among immunocompromised patients with acute respiratory failure: a randomized clinical trial. JAMA 2015;314(16):17111719.

11. Nishimura M. High-flow nasal cannula oxygen therapy in adults: physiological benefits, indication, clinical benefits, and adverse effects. Respir Care 2016;61(4):529-541.

12. Helviz Y, Einav S. A systematic review of the high-flow nasal cannula for adult patients. Crit Care 2018;22(1):71.

13. Frat JP, Thille AW, Mercat A, Girault C, Ragot S, Perbet S, et al. High-flow oxygen through nasal cannula in acute hypoxemic respiratory failure. N Engl J Med 2015;372(23):2185-2196.

14. Mauri T, Turrini C, Eronia N, Grasselli G, Volta CA, Bellani G, et al. Physiologic effects of high-flow nasal cannula in acute hypoxemic respiratory failure. Am J Respir Crit Care Med 2017;195(9):12071215.

15. Spoletini G, Alotaibi M, Blasi F, Hill NS. Heated humidified highflow nasal oxygen in adults: mechanisms of action and clinical implications. Chest 2015;148(1):253-261.

16. De Jong A, Calvet L, Lemiale V, Demoule A, Mokart D, Darmon M, et al. The challenge of avoiding intubation in immunocompromised patients with acute respiratory failure. Expert Rev Respir Med 2018; 12(10):867-880.

17. Frat JP, Coudroy R, Marjanovic N, Thille AW. High-flow nasal oxygen therapy and noninvasive ventilation in the management of acute hypoxemic respiratory failure. Ann Transl Med 2017;5(14): 297.

18. Sklar MC, Mohammed A, Orchanian-Cheff A, Del SL, Mehta S, Munshi L. The impact of high-flow nasal oxygen in the immunocompromised critically ill: a systematic review and meta-analysis. Respir Care 2018;63(12):1555-1566.

19. Huang HB, Peng JM, Weng L, Liu GY, B D. High-flow oxygen therapy in immunocompromised patients with acute respiratory failure: a review and meta-analysis. J Crit Care 2018;43:300-305.

20. Cortegiani A, Crimi C, Sanfilippo F, Noto A, Di Falco D, Grasselli $\mathrm{G}$, et al. High flow nasal therapy in immunocompromised patients with acute respiratory failure: a systematic review and meta-analysis. J Crit Care 2019;50:250-256.

21. Azoulay E, Lemiale V, Mokart D, Nseir S, Argaud L, Pene F, et al. Effect of high-flow nasal oxygen vs standard oxygen on 28-day mortality in immunocompromised patients with acute respiratory failure: the HIGH randomized clinical trial. JAMA 2018;320(20): 2099-2107.

22. Rochwerg B, Granton D, Wang DX, Helviz Y, Einav S, Frat JP, et al. High flow nasal cannula compared with conventional oxygen therapy for acute hypoxemic respiratory failure: a systematic review and meta-analysis. Intensive Care Med 2019;45(5):563-572.

23. Higgins JP, Altman DG, Gotzsche PC, Juni P, Moher D, Oxman AD, et al. The Cochrane Collaboration's tool for assessing risk of bias in randomised trials. BMJ 2011;343:d5928.

24. Stang A. Critical evaluation of the Newcastle-Ottawa scale for the assessment of the quality of nonrandomized studies in meta-analyses. Eur J Epidemiol 2010;25(9):603-605. 


\section{HFNC IN IMMUNOCOMPROMISED SUbJECTS With ARF}

25. Higgins JP, Thompson SG, Deeks JJ, Altman DG. Measuring inconsistency in meta-analyses. BMJ 2003;327(7414):557-560.

26. Tu G, He H, Yin K, Ju M, Zheng Y, Zhu D, et al. High-flow nasal cannula versus noninvasive ventilation for treatment of acute hypoxemic respiratory failure in renal transplant recipients. Transplant Proc 2017;49(6):1325-1330.

27. Lemiale V, Resche-Rigon M, Mokart D, Pène F, Argaud L, Mayaux $\mathrm{J}$, et al. High-flow nasal cannula oxygenation in immunocompromised patients with acute hypoxemic respiratory failure: a groupe de recherche respiratoire en réanimation onco-hématologique study. Crit Care Med 2017;45(3):e274-e280.

28. Coudroy R, Jamet A, Petua P, Robert R, Frat JP, Thille AW. Highflow nasal cannula oxygen therapy versus noninvasive ventilation in immunocompromised patients with acute respiratory failure: an observational cohort study. Ann Intensive Care 2016;6(1):45.

29. Frat JP, Ragot S, Girault C, Perbet S, Prat G, Boulain T, et al. Effect of non-invasive oxygenation strategies in immunocompromised patients with severe acute respiratory failure: a post-hoc analysis of a randomised trial. Lancet Respir Med 2016;4(8):646-652.

30. Lemiale V, Mokart D, Mayaux J, Lambert J, Rabbat A, Demoule A, et al. The effects of a 2-h trial of high-flow oxygen by nasal cannula versus Venturi mask in immunocompromised patients with hypoxemic acute respiratory failure: a multicenter randomized trial. Crit Care 2015;19:380.

31. Roca O, de Acilu MG, Caralt B, Sacanell J, Masclans JR. Humidified high flow nasal cannula supportive therapy improves outcomes in lung transplant recipients readmitted to the intensive care unit because of acute respiratory failure. Transplantation 2015;99(5):10921098.

32. Azoulay E, Schellongowski P, Darmon M, Bauer PR, Benoit D, Depuydt $\mathrm{P}$, et al. The Intensive Care Medicine research agenda on critically ill oncology and hematology patients. Intensive Care Med 2017;43(9):1366-1382.

33. Brochard L, Slutsky A, Pesenti A. Mechanical ventilation to minimize progression of lung injury in acute respiratory failure. Am J Respir Crit Care Med 2017;195(4):438-442.

34. Ni YN, Luo J, Yu H, Liu D, Ni Z, Cheng J, et al. Can high-flow nasal cannula reduce the rate of endotracheal intubation in adult patients with acute respiratory failure compared with conventional oxygen therapy and noninvasive positive pressure ventilation? A systematic review and meta-analysis. Chest 2017;151(4):764-775.

35. Ou X, Hua Y, Liu J, Gong C, Zhao W. Effect of high-flow nasal cannula oxygen therapy in adults with acute hypoxemic respiratory failure: a meta-analysis of randomized controlled trials. CMAJ 2017; 189(7):E260-E267.

36. Moller W, Feng S, Domanski U, Franke KJ, Celik G, Bartenstein P, et al. Nasal high flow reduces dead space. J Appl Physiol (1985) 2017;122(1):191-197.
37. Nishimura M. High-flow nasal cannula oxygen therapy in adults. J Intensive Care 2015;3(1):15.

38. Parke RL, Bloch A, McGuinness SP. Effect of very-high-flow nasal therapy on airway pressure and end-expiratory lung impedance in healthy volunteers. Respir Care 2015;60(10):1397-1403.

39. Nielsen KR, Ellington LE, Gray AJ, Stanberry LI, Smith LS, DiBlasi RM. Effect of high-flow nasal cannula on expiratory pressure and ventilation in infant, pediatric, and adult models. Respir Care 2018; 63(2):147-157.

40. Vargas F, Saint-Leger M, Boyer A, Bui NH, Hilbert G. Physiologic effects of high-flow nasal cannula oxygen in critical care subjects. Respir Care 2015;60(10):1369-1376.

41. Goligher EC, Slutsky AS. Not just oxygen? Mechanisms of benefit from high-flow nasal cannula in hypoxemic respiratory failure. Am J Respir Crit Care Med 2017;195(9):1128-1131.

42. Barbas CS, Serpa NA. New puzzles for the use of non-invasive ventilation for immunosuppressed patients. J Thorac Dis 2016;8(1): E100-E103.

43. Luo J, Wang MY, Zhu H, Liang BM, Liu D, Peng XY, et al. Can non-invasive positive pressure ventilation prevent endotracheal intubation in acute lung injury/acute respiratory distress syndrome? A meta-analysis. Respirology 2014;19(8):1149-1157.

44. Spoletini G, Cortegiani A, Gregoretti C. Physiopathological rationale of using high-flow nasal therapy in the acute and chronic setting: a narrative review. Trends Anaesth Crit Care 2019;26-27:22-29.

45. Frat JP, Ragot S, Coudroy R, Constantin JM, Girault C, Prat G, et al. Predictors of intubation in patients with acute hypoxemic respiratory failure treated with a noninvasive oxygenation strategy. Crit Care Med 2018;46(2):208-215.

46. Marshall JC, Cook DJ, Christou NV, Bernard GR, Sprung CL, Sibbald WJ. Multiple organ dysfunction score: a reliable descriptor of a complex clinical outcome. Crit Care Med 1995;23(10):1638-1652.

47. Frat JP, Coudroy R, Thille AW. Non-invasive ventilation or highflow oxygen therapy: when to choose one over the other? Respirology 2018

48. Cortegiani A, Crimi C, Noto A, Helviz Y, Giarratano A, Gregoretti C, et al. Effect of high-flow nasal therapy on dyspnea, comfort, and respiratory rate. Crit Care 2019;23(1):201.

49. Kangelaris KN, Ware LB, Wang CY, Janz DR, Zhuo H, Matthay MA, et al. Timing of intubation and clinical outcomes in adults with acute respiratory distress syndrome. Crit Care Med 2016;44(1):120129.

50. Sztrymf B, Messika J, Bertrand F, Hurel D, Leon R, Dreyfuss D, et al. Beneficial effects of humidified high flow nasal oxygen in critical care patients: a prospective pilot study. Intensive Care Med 2011;37(11):1780-1786.

51. Chiumello D, Brochard L, Marini JJ, Slutsky AS, Mancebo J, Ranieri VM, et al. Respiratory support in patients with acute respiratory distress syndrome: an expert opinion. Crit Care 2017;21(1):240. 